Journal of ISMAC (2019)

Vol.01/ No. 03

Pages: 147-159

http://irojournals.com/iroismac/

DOI: https://doi.org/10.36548/jismac.2019.3.002

\title{
A COMPREHENSIVE SURVEY ON THE COMPUTATIONAL INTELLIGENCE TECHNIQUES AND ITS APPLICATIONS
}

\author{
Dr. Jennifer S. Raj, \\ Professor, \\ Department of ECE, Gnanamani College of Technology, \\ Namakkal, India. \\ Email: jennifer.raj@gmail.com
}

\begin{abstract}
The artificial intelligence that tries to imitate the human beings by gathering a vast knowledge gained using the reasoning, planning, searching and prediction fails in certain areas that necessitate a construction of large set of rules. The AI also faces challenges due to the growing demands in the learning and the search optimization. These failures of AI paved a path for the growth of the computational tools that led to the rise of the new regimen that is the computational intelligence. The paper presents the comprehensive survey of the computational intelligent techniques and its applications as they seem to be an effective alternative for the artificial intelligence overcoming the failures and the draw backs in it.
\end{abstract}

Keywords: Artificial intelligence, Computational techniques, Computational intelligence, Fuzzy logic, Neural networks, Evolutionary algorithm, Learning theory, Probabilistic method

\section{INTRODUCTION}

Though the artificial intelligence and the computational intelligence almost scopes for the same objective, they are primarily different. The artificial intelligence imitates the intelligence of the human beings in the machines. It is boundless regimen comprised vast insights that incorporates learning, reasoning, planning, intelligent searching and perception building.

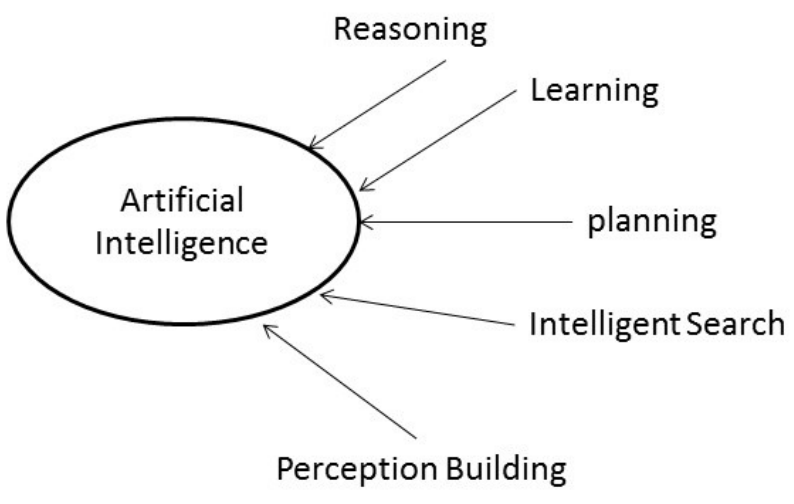

ISSN: 2582-1369 (online) 
Journal of ISMAC (2019)

Vol.01/ No. 03

Pages: 147-159

http://irojournals.com/iroismac/

DOI: https://doi.org/10.36548/jismac.2019.3.002

Fig .1 Insights of Artificial Intelligence

The fig. 1 shows the methods that are utilized in gaining the knowledge. The reasoning solves a problem using the knowledge that is pre-defined. Learning can be described as the encoding process that encodes the conditions and connected and stores it into the memory to recall it when necessary. Planning solves a problem by determining the sequence of steps entailed in solving a problem. Many of the traditional problems that were dealt by the artificial intelligence were often expressed as the state-space search. The space is usually the collection of the states and the state is the particular instance. The flow chart in the fig .2 below explains the intelligent search through the artificial intelligence.



Fig .2 Intelligent Search

But the traditional artificial intelligence faced multiple problems due to the construction of large set of rules, and the transition of the states. All these make the conventional AI incompatible for the growing demands in the search based optimization and the learning as the data base available is very high. The failures of the conventional AI paved way for the emergence of the computational tools that led to the rise of the new regimen called the computational intelligence [1] the fig. 4 below shows the computational tools.

ISSN: 2582-1369 (online) 
Journal of ISMAC (2019)

Vol.01/ No. 03

Pages: 147-159

http://irojournals.com/iroismac/

DOI: https://doi.org/10.36548/jismac.2019.3.002

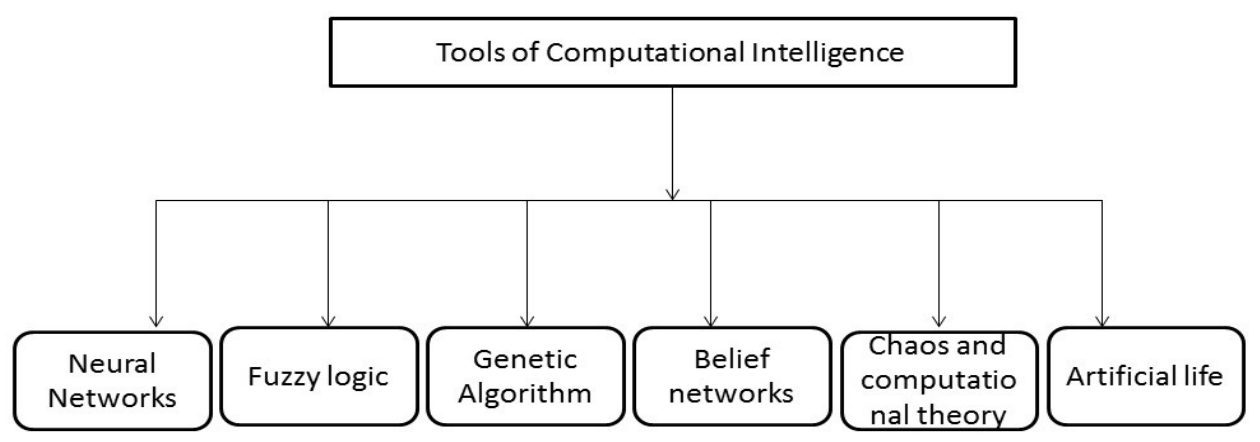

Fig .3 Tools of Computational Intelligence

It is roughly defined as the "system dealing with the low level data, including the pattern recognition components to exhibit computational -adaptivity, computational-fault tolerance with the human like performance in speed and the approximation of the error rates, Were the computational adaption refers to the system capability to adapt to the temporal changes in the data provided at the input and that achieved at the output. The configurations in the parallel manner enable the fault tolerance in the computations even if the there is a failure in any one of the units. Few e.g. computational tools with the fault tolerance are the artificial neural networks and the Fuzzy logic. The other tools can be arranged in the distributed manner to achieve the fault tolerance in them. All these merits make the computational intelligence more suitable for the present day for the having optimal solution for the problems.

So the paper is to survey the computational intelligence techniques and the applications related to it.

The paper remaining is organized with the section 2 providing the related work based on the survey with the techniques used in the computational intelligence and its applications and section 3 describing the techniques in the computational intelligence 4. Provides the applications and the summarization of the survey. 5. Conclusion

\section{RELATED WORKS}

Konar et al [1] the author details the emergence of the computational intelligence as an alternative to the traditional artificial intelligence to overcome the incompatibility of the artificial intelligence that is conventional in satisfying

ISSN: 2582-1369 (online) 
Journal of ISMAC (2019)

Vol.01/ No. 03

Pages: 147-159

http://irojournals.com/iroismac/

DOI: https://doi.org/10.36548/jismac.2019.3.002

the growing demands in the search optimization and the machine learning. The insufficiency of conventional artificial intelligence was more and more recognized due to the continuous failures in the fifth generation computers that were developed by the Japanese. In this paper the author describes the principles, techniques and the applications of the computational intelligence.

Rutkowski, et al [2] the author presents the computational intelligence as the strategy to solve the problems evolving in the artificial intelligence utilizing the numerical calculations. He further elaborates the techniques used for the computation as the neural networks, fuzzy logic, evolutionary algorithms, rough sets, uncertain variables, probabilistic methods. The techniques mention above are either utilized in single or hybrid forms to solve the complex issues of the artificial intelligence in the processing of the speech, natural languages, for structuring of the expert systems and the robots.

Ansari, A. Q. et al [3] the author explains the fuzzy logic basics; he describes the fuzzy as a highly powerful tool in dealing with the complex problems. The author states that the fuzzy logic can be framed to characterize the and control a system whose model is under disguise or ill defined. He further describes the fuzzy logic abilities in processing the natural statement depicting the reasoning and the decision making of the human common sense.

Siddique et al [4] the author details the basics of the fuzzy logic along with the detailed description on the basics of the neuro-computing and the neural systems. He presents neuro-computing as the highly significant concepts that is based on the functioning of the brain and could be applied with a wide range of applications that are used in the system identification, simulation and the adaption. And also presents the details of the evolutionary, learning theory and the probabilistic methods.

Rumelhart, et al [5] the author presents a study on the neural networks that could be utilized in solving the complex problems. The learning based on the concept of the neural network and the back propagation is examined in the paper.

Alavala et al [6] the book provides the comprehensive insights on the fundamentals of the fuzzy logic and the neural networks, along with the details of the fuzzy logic and the neural network.

ISSN: 2582-1369 (online) 
Journal of ISMAC (2019)

Vol.01/ No. 03

Pages: 147-159

http://irojournals.com/iroismac/

DOI: https://doi.org/10.36548/jismac.2019.3.002

Alatas, et al [7] the insufficiency in the traditional and the conventional algorithms have been overcome by the introduction of the computational intelligence in the global optimization problem that proceeds with the aim of finding a more optimized solutions.

Chouhan et al [8] the author details the image segmentation process that is the preprocessing phase to gather valuable insight from the images utilizing the computational techniques such as the neural network, fuzzy logic, genetic algorithm. The author presents this as a review focusing on the various methodologies of the computational intelligence with the multitude of applications on the image segmentation.

Akhani, et al [9] the author present a hybridized frame work of the evolutionary algorithm involving the genetic algorithm, neural networks and the regression analysis for the prediction of the spectral acceleration attributes of a strong ground- movements.

Tareen et al [10] the paper proceeds with the computational intelligence for the automated anomalous behavior detection in the soil radon with the accuracy of the models being enumerated using the MAE, RMS and the MSE.

Tariq et al [11] the involvement of the computational intelligence in the petroleum engineering applications for the accurate predictions of the FBHP (flowing bottom-hole pressure) in the vertical well that is naturally flowing with the multiphase flow is presented in the paper.

Shahid et al [12] the author presents the computational intelligence as the knowledge acquisition strategies in the medical diagnosis and the prognosis for the important decision making. The author also discusses the features of the computational intelligence techniques in the medical diagnosis providing a new awareness for the enhancement of the research's in the future for the domain of medical diagnosis.

Neelima et al [13] a short analysis of the intrusion detection applying various computational intelligence techniques such as the fuzzy logic, neural network, machine learning, evolutionary algorithm, and probabilistic reasoning is presented in the paper along with the future challenges, advantages, and the limitations.

Alippi et al [14] the author describes the usage of the computational intelligence in the period of the cyber-physical systems and the internet of things.

ISSN: 2582-1369 (online) 
Journal of ISMAC (2019)

Vol.01/ No. 03

Pages: 147-159

http://irojournals.com/iroismac/

DOI: https://doi.org/10.36548/jismac.2019.3.002

Kumar et al [15] the paper is a review about the computational techniques employed in the biometric recognition.

Chen et al [16] the author addresses the computational intelligence as the one of the fast developing regimen in the computer technology and details the importance of the computational intelligence in the application of the exigent situations in the present days.

\section{COMPUTATION INTELLIGENCE TECHNIQUES}

The computational intelligence could be defined as the "combination of the intelligent tools and the computational models that are capable of directly accepting a raw data directly and process them directly in a distributed manner for producing of periodic responses are highly fault tolerant" the block below explains the process involved in the computational intelligence [1].

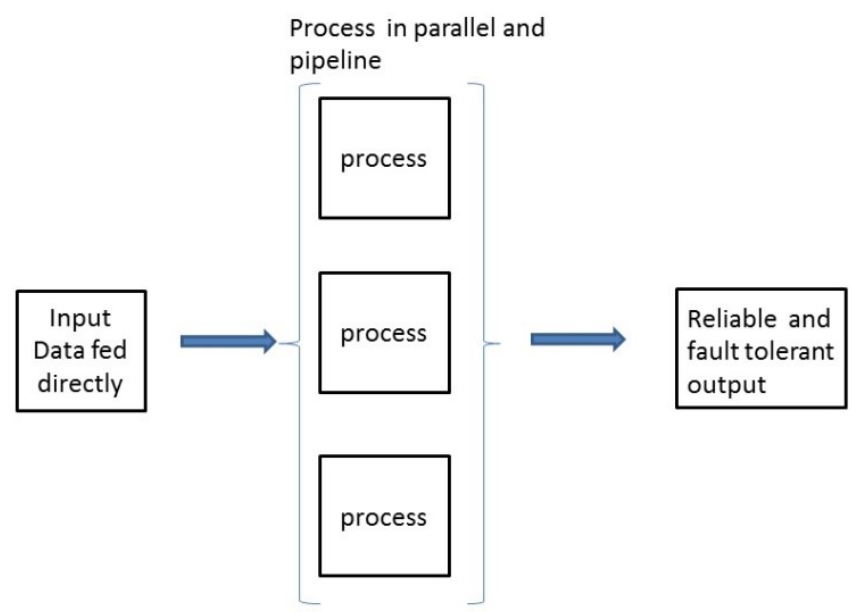

Fig.4 Computational Intelligence

With the evolution of the soft computing the computational intelligence was described as the subset of the AI and in the two types of machine intelligence, the intelligent that was based on the hard computing was called the AI and one that was based on the soft computing is called the CI the section below details the principle techniques of the computational intelligence. 
Journal of ISMAC (2019)

Vol.01/ No. 03

Pages: 147-159

http://irojournals.com/iroismac/

DOI: https://doi.org/10.36548/jismac.2019.3.002

3.1. FUZZY LOGIC: The concept of the fuzzy logic was proposed in 1965 by the lofti zadeh, the fuzzy set is often considered to be the super set of the Boolean logic. In Boolean logic all the data's can be represented only using two values either the 'zero' or the 'one'. The data given as the input either falls under the state 'zero' representing the logic low or the state 'one' denoting the logic high. A threshold is set and the value below the threshold is defined as the logic high and the one that is below is defines as the logic low. But this not in the case in the fuzzy logic, the fuzzy logic defines the multiple states that lies in between the high and the low levels. For a given range of inputs ranging from 0 to 1 the fuzzy set could be defined as the all the intermediate number that lie between the zero to one and all the values in them that describe the degree of the truth could be denoted as the member ship function $M$. Where $M$ is mathematically expressed as the $0<M<1$. The figure. 5 below shows the membership functions for the true and the false values in selecting the frequency of the radio station that is 103.5

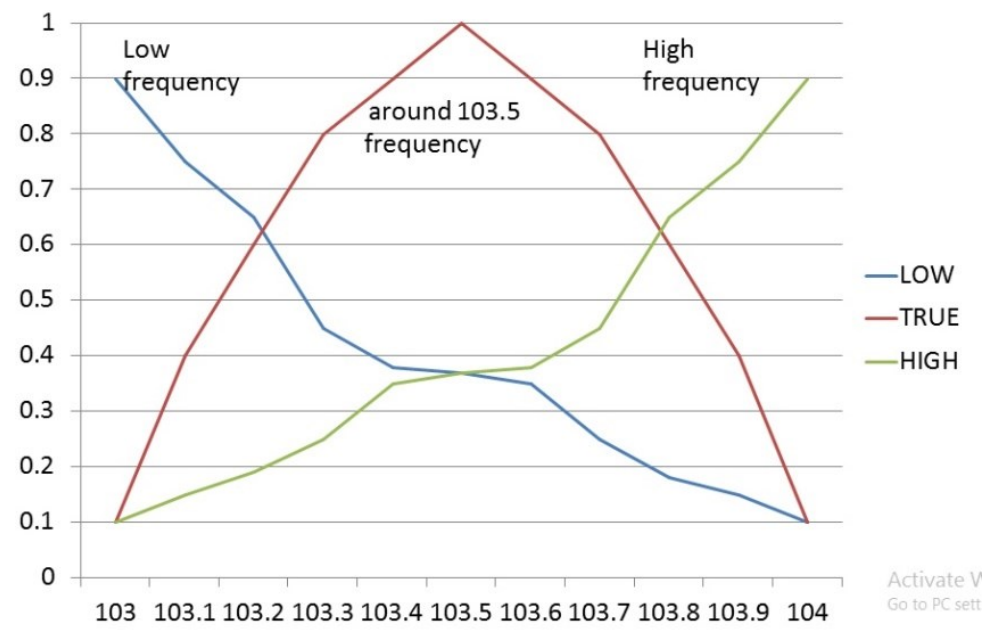

Fig .5Membership Functions

From the fig. 5 it is understood that the high and the low values denote the non-members of the fuzzy sets, and the true values show they are the members of the fuzzy set. The curve around the frequencies 103.5 can be expressed mathematically as the $F=\sum_{S} M F(X) / X$, where the summation just denotes the collection of the true values. The hedges are used in denoting the extremely low frequencies and the low frequencies. Very low is denoted as the $M^{2}$, extremely low is denoted as the $M^{3}$, and the in between cases fall in the ranges of $M^{.5}$.the fuzzy set could be applied with any applications that has numerous of states to be evaluated between the values that are members and nonmembers.[3] [4] 
Journal of ISMAC (2019)

Vol.01/ No. 03

Pages: 147-159

http://irojournals.com/iroismac/

DOI: https://doi.org/10.36548/jismac.2019.3.002

3.2. NEURAL NETWORKS: The neural networks [5] [6] that is based on the concept of the computation that are similar to the brain, is considered in variety of ways such as the "parallel distributed processing, artificial neural system, neuromorphic computation" the ensure a computation in a parallel manner providing a speedy processing that is unlike the traditional computer that work in the serial manner taking much time for the computations. The basic concept of the neural networks is to design a simple mathematical frame works that resemble the brain system and proceed to learn them and get to know the device capability in solving the various complex problems. The Fig. 6 below shows the steps involved in the neural networks.

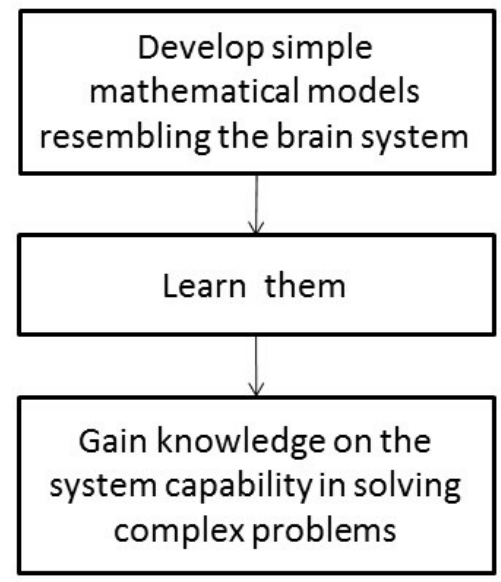

Fig .6 Concept of Neural Network

So the neural networks are capable of working like humans with fault tolerance. The neural networks can be associated with multitudes of applications that necessitate the data analysis, clustering, associative memory, classifications, and in the generation of the patterns and the controls. The most application that rely on the neural networks are the medical information's that require a classification, intrusion and fraud detection etc. The Siddique et al [4] insists that the neural network can also be hybridized with any other techniques of the CI to have a better solution to the complex problem.

3.3. EVOLUTIONARY ALGORITHM: The fundamental concept of the evolutionary algorithm lays back on the procedures of the natural selection. They are very well preferred in the areas where the conventional mathematical methods are incompatible for a broader range of problems and usually engaged in the applications such as the DNA analysis and the scheduling problems. One of the prominent evolution algorithms that are based on the process of the natural selection is the genetic algorithm that takes through the steps of the initializing population and evaluating

ISSN: 2582-1369 (online) 
Journal of ISMAC (2019)

Vol.01/ No. 03

Pages: $147-159$

http://irojournals.com/iroismac/

DOI: https://doi.org/10.36548/jismac.2019.3.002

the optimal one with the help of mutation and cross over. These evolutionary algorithms aim in bringing out novel artificial evolutionary techniques exploiting the strength of the natural evolution and are most probably engaged in the search optimization problems that requires an optimal results. The fig. 7 below shows the procedures of genetic algorithm

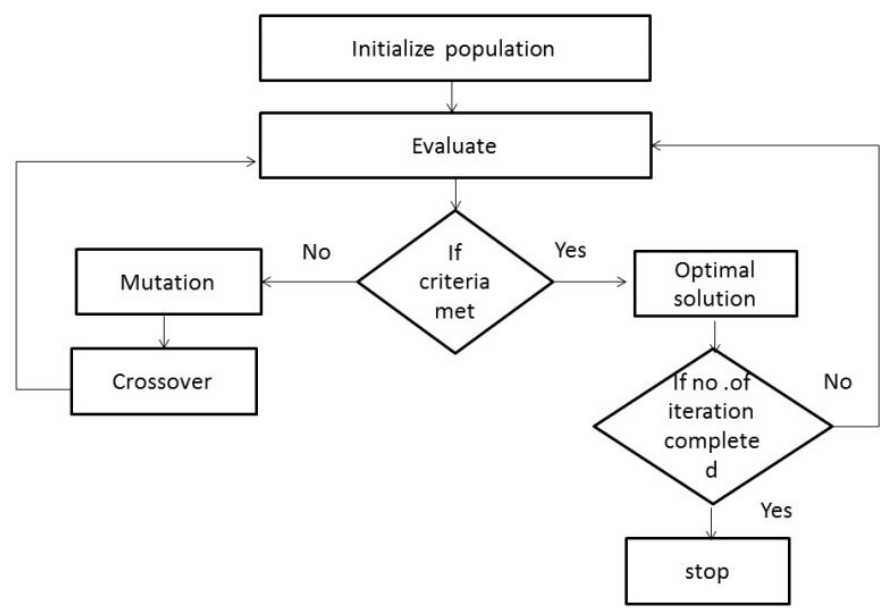

Fig. 7 Concept of Genetic Algorithm

3.4. LEARNING THEORY: It is one of the significant approaches of the Computational intelligence. The learning theory enables to grasp the effect and the experiences of an event and acquire deep insights from them and utilize them to predict the future results with the help of the previous experiences gained. The most popular learning in the present day is the machine learning [1] which could categorized as the supervised, unsupervised and the reinforcement learning. The gather the information from the previous events that has occurred learn them and utilize them in the predicting the accurate output for the problem. Each time they analyze the problem they match it with the previous occurrences that are been learned to produce accurate results. These types of computational intelligence are usually engaged in the application that requires diagnoses, detection and prediction. So the learning theory could be further classified into 5 types with the learning's based on the behaviorism, constructivism, Cognitivism, experientialism and the social learning. The fig. 8 below shows the five bases of learning theory. 
Journal of ISMAC (2019)

Vol.01/ No. 03

Pages: $147-159$

http://irojournals.com/iroismac/

DOI: https://doi.org/10.36548/jismac.2019.3.002

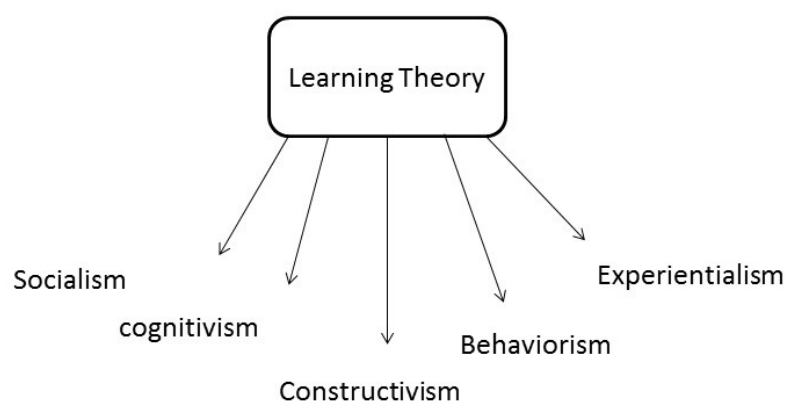

Fig .8 Learning Theories

3.5. PROBABILISTIC METHODS: They are significant element on the fuzzy logic. It is referred as a nonconstructive method and initially engaged in the mathematical area that is primarily concerned with the counting. It enumerates the outputs of the intelligent systems that are based on the computational intelligence in a random way in order to determine the probable results for the problem that are based on the insight acquired in prior.

\section{APPLICATIONS}

This section presents the application of the computational intelligence in the various fields for the accurate result prediction, and the analysis. The efficiency of the computational intelligence with the speedy processing and the accuracy in the finding the results makes it more popular among various applications such as the intrusion detection [13] [14], global optimization [7], image segmentation [8] spectral acceleration prediction [9], automated anomalous behavior detection [10], liquid flow prediction [11] and in medical diagnosis [12], biometric detection [15], emergency management [16] and even more. The challenges faced in the CI in terms of the accuracy, reasoning, diagnoses, detection and the predictions can be even manipulated applying the hybridized computational techniques combining the tools of the computation [7] [9] the tabulation below in the table.1 presents the information gathered from different literatures related to the survey. 
Journal of ISMAC (2019)

Vol.01/ No. 03

Pages: $147-159$

http://irojournals.com/iroismac/

DOI: https://doi.org/10.36548/jismac.2019.3.002

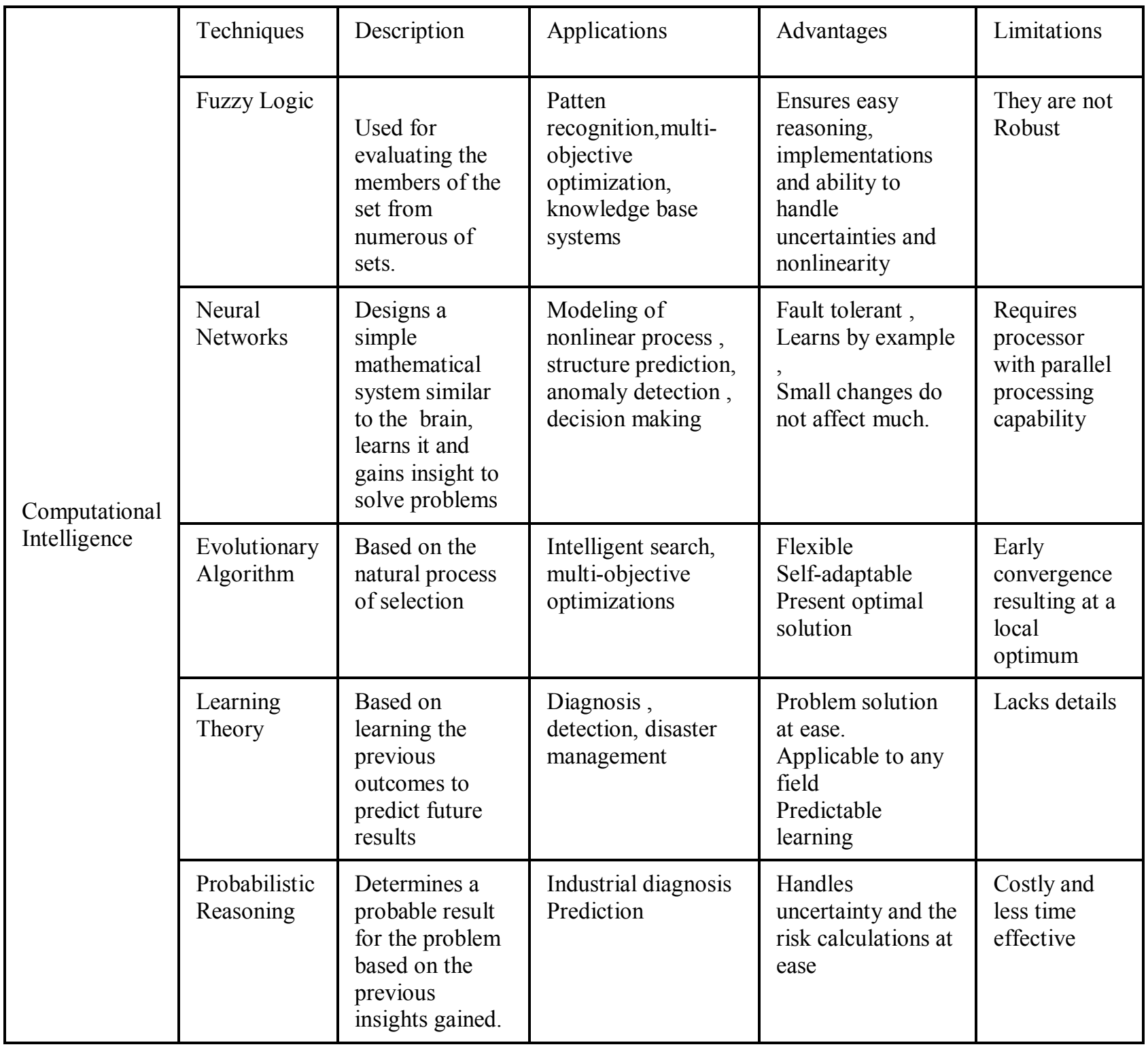

Table .1 Summarization of the Survey

\section{CONCLUSION}

The comprehensive survey on the computational intelligence techniques that are renowned for its speedy process and accurate results prediction along with the its applications are presented in the paper detailing the importance of the computational intelligence and its necessity as an alternative to the traditional artificial intelligence that shows draw backs in certain cases of search optimization and the learning. The paper further continues with the

ISSN: 2582-1369 (online) 
Journal of ISMAC (2019)

Vol.01/ No. 03

Pages: 147-159

http://irojournals.com/iroismac/

DOI: https://doi.org/10.36548/jismac.2019.3.002

descriptions on the techniques of the computational intelligence and the applications that benefits from them. In future, the paper proceeds with the analysis of the various computational techniques to find the optimal one in the detection of the fraudulent access.

\section{References}

[1] Konar, Amit. Computational intelligence: principles, techniques and applications. Springer Science \& Business Media, 2006.

[2] Rutkowski, Leszek. Computational intelligence: methods and techniques. Springer Science \& Business Media, 2008.

[3] Ansari, A. Q. "The basics of fuzzy logic: A tutorial review." COMPUTER EDUCATION-STAFFORDCOMPUTER EDUCATION GROUP- 88 (1998): 5-8.

[4] Siddique, Nazmul, and Hojjat Adeli. Computational intelligence: synergies of fuzzy logic, neural networks and evolutionary computing. John Wiley \& Sons, 2013.

[5] Rumelhart, David E., Bernard Widrow, and Michael A. Lehr. "The basic ideas in neural networks." Communications of the ACM 37, no. 3 (1994): 87-93.

[6] Alavala, Chennakesava R. Fuzzy logic and neural networks: basic concepts \& application. New Age International, 2008.

[7] Alatas, Bilal. "Sports inspired computational intelligence algorithms for global optimization." Artificial Intelligence Review 52, no. 3 (2019): 1579-1627.

[8] Chouhan, Siddharth Singh, Ajay Kaul, and Uday Pratap Singh. "Image segmentation using computational intelligence techniques." Archives of Computational Methods in Engineering 26, no. 3 (2019): 533-596.

[9] Akhani, Mohsen, Ali R. Kashani, Mehdi Mousavi, and Amir H. Gandomi. "A hybrid computational intelligence approach to predict spectral acceleration." Measurement 138 (2019): 578-589.

[10] Tareen, Aleem Dad Khan, Khawaja M. Asim, Kimberlee Jane Kearfott, Muhammad Rafique, Malik Sajjad Ahmed Nadeem, Talat Iqbal, and Saeed Ur Rahman. "Automated anomalous behaviour detection in soil radon gas prior to earthquakes using computational intelligence techniques." Journal of environmental radioactivity 203 (2019): 48-54.

[11] Tariq, Zeeshan, Mohamed Mahmoud, and Abdulazeez Abdulraheem. "Real-time prognosis of flowing bottom-hole pressure in a vertical well for a multiphase flow using computational intelligence techniques." Journal of Petroleum Exploration and Production Technology (2019): 1-18.

[12] Shahid, Afzal Hussain, and M. P. Singh. "Computational intelligence techniques for medical diagnosis and prognosis: Problems and current developments." Biocybernetics and Biomedical Engineering (2019). 
Journal of ISMAC (2019)

Vol.01/ No. 03

Pages: $147-159$

http://irojournals.com/iroismac/

DOI: https://doi.org/10.36548/jismac.2019.3.002

[13] Neelima, D., J. Karthik, K. Aravind John, S. Gowthami, and Janmenjoy Nayak. "Soft Computing-Based Intrusion Detection Approaches: An Analytical Study." In Soft Computing in Data Analytics, pp. 635-651. Springer, Singapore, 2019.

[14] Alippi, Cesare, and Seiichi Ozawa. "Computational Intelligence in the Time of Cyber-Physical Systems and the Internet of Things." In Artificial Intelligence in the Age of Neural Networks and Brain Computing, pp. 245-263. Academic Press, 2019.

[15] Kumar, Mrityunjay, and Arvind Kumar Tiwari. "Computational Intelligence Techniques for Biometric Recognition: A Review." Available at SSRN 3350260 (2019).

[16]Chen, Ning, Wenjing Liu, Ruizhen Bai, and An Chen. "Application of computational intelligence technologies in emergency management: a literature review." Artificial Intelligence Review 52, no. 3 (2019): 2131-2168. 